\title{
DETERMINANTES E DIFICULDADES DE CRESCIMENTO PARA MULHERES EMPREENDEDORAS
}

\section{DETERMINANTS AND DIFFICULTIES OF ENTERPRISES GROWTH BY WOMEN ENTREPRENEURS}

\author{
Recebido em 10.03.2016. Aprovado em 16.03.2017 \\ Avaliado pelo sistema double blind review \\ DOI: http://dx.doi.org/10.12712/rpca.v11i1.828
}

\section{Hilka Pelizza Vier Machado \\ hilkavier@yahoo.com \\ Universidade do Oeste de Santa Catarina (UNOESC), Joaçaba/SC, BRASIL \\ Centro Universitário de Maringá (UNICESUMAR), Maringá/PR, BRASIL}

\section{Alexandre Guedes \\ adm.guedes@gmail.com \\ Instituto Adventista Paranaense, Ivatuba/PR, BRASIL}

\section{Sebastião Gazola}

sgazola@uem.br

Universidade Estadual de Maringá(UEM), Maringá/PR, BRASIL

\section{Resumo}

O objetivo desta pesquisa foi identificar determinantes e dificuldades de crescimento de empresas de mulheres, bem como a relação de características da empresa e das empreendedoras com estas variáveis. A pesquisa foi realizada com uma amostra de 102 empreendedoras. O instrumento para coleta de dados foi um questionário estruturado. O tratamento dos dados foi realizado com análises descritivas e exploratórias. O teste de Spearman mostrou a correlação entre as determinantes e dificuldades de crescimento com características da empresa e das empreendedoras. A análise de Cluster mostrou grupos de atributos similares relacionados a determinantes e dificuldades de crescimento. As principais determinantes foram: tempo dedicado ao negócio, conhecimento prévio, capacidade criativa e inovadora e novos produtos e serviços. As principais dificuldades foram falta de qualificação pessoal, falta de autoconfiança, baixa capacidade inovadora; falta de planejamento, falta de organização, falta de informações sobre o negócio e falta de estratégia de crescimento.

Palavras-chave: Mulheres Empreendedoras. Empreendedorismo. Crescimento de Empresas.

\section{Abstract}

The aim of this research was to identify determinants and difficulties encountered by women entrepreneurs, as well as the relationship with characteristics of the enterprise and of the women entrepreneurs. This research was conducted join a 102 women entrepreneurs. The instrument for data collection was a structured questionnaire. Descriptive and exploratory analyses were employed in the data analysis. The test of Spearman showed the correlation between determinant and growth difficulties and characteristics of the company and characteristics of the entrepreneurs. The analysis of Cluster identified groups of similar attributes related to the determinant and the growth difficulties. The main determinants were: time dedicated to the business, previous knowledge, creative capacity and innovative and new products and services. The growth difficulties were lack of personal qualification, lack of self trust and not to be innovative; planning lack, lack of organization, lack of information on the business and lack of growth strategy.

Keywords: Women Entrepreneurs. Entrepreneurship. Growth of Enterprises. 


\section{Introdução}

A criação de empresas representa o início do processo empreendedor, mas a perspectiva processual do Empreendedorismo requer também a compreensão do crescimento, assim como da sobrevivência ou não do empreendimento.

No tocante ao crescimento, este tem suscitado recentemente interesse por parte de pesquisadores que têm investigado o fenômeno para além da abordagem da quantidade, na medida em que o crescimento pode abranger múltiplas dimensões, como por exemplo, a satisfação do cliente e dos empregados, ou o desempenho social e ambiental (SANTOS; BRITO, 2012). Desta forma, explicações contextuais, sociais e mais abrangentes do crescimento precisam ser buscadas (CLARKE et al, 2014).

O crescimento é um fenômeno complexo e heterogêneo, principalmentepelos seus modos diversos de interpretação (DAVIDSSON et al., 2010; DOBBS; HAMILTON, 2007). Além disso, variações em taxas de crescimento podem ser identificadas ao longo de diferentes períodos (BRITO; VASCONCELOS, 2009) e podem ser influenciadas pelo contexto de negócio ou pelas expectativas de empreendedores (DELMAR et al., 2003; DAVIDSSON et al., 2010; ACHTENHAGEN et al., 2010). No que se refere à expectativa de empreendedores, especialmente em pequenos negócios, a determinação do ritmo e de percentuais de crescimento dependem de intenções e motivações de empreendedores (DAVIDSSON et al., 2010). Isso porque empreendedores apresentam diferentes intenções e aspirações de crescimento e, em seqüência, delineiam estratégias para promoverem o crescimento de seus negócios (MITCHELMORE; ROWLEY, 2013).

Esses aspectos evidenciam a necessidade de se analisar o crescimento sob um enfoque processual, que implica na compreensão de antecedentes e conseqüentes, abrangendo, por exemplo, estudos sobre intenções, objetivos, planejamento, estratégias e medidas de crescimento (COSTIN, 2012). Contudo, esses aspectos não abrangem o processo de forma integral, na medida em que empreendedores enfrentam dificuldades para promover o crescimento de seus negócios e que alguns elementos podem ser determinantes para o crescimento. Estas variáveis, ou seja, dificuldades e determinantes de crescimento representam parte do processo de crescimento que foram estudadas nesta pesquisa.
Para estudar determinantes e dificuldades de crescimento, optou-se por realizar um estudo junto a mulheres empreendedoras, tendo em vista que estudos anteriores constataram que empresas de homens crescem mais do que as de mulheres (COAD; TAMVADA, 2012) e que mulheres são menos propensas a apresentar expectativas de elevado crescimento (ARROYO et al., 2016). Em geral, os negócios de mulheres permanecem pequenos em termos de lucros, vendas e de número de empregados (MORRIS et al., 2006). No entanto, estudos anteriores apontaram que o reduzido crescimento não se deve à falta de ambição de crescimento ou de intenção de crescimento, pois esta foi identificada entre empreendedoras, desde o momento da criação da empresa, conforme salientaram Dalborg et al. (2012) e Machado et al. (2016). Com isso, outros fatores restringem o crescimento dessas empresas e precisam ser investigados, na medida em que mulheres estão, cada vez mais, criando empresas (LERNER; ALMOR, 2002; ALSOS et al., 2006; BAUGHAN et al., 2006).

Outros estudos sobre crescimento de empresas de mulheres exploraram parcialmente aspectos do processo de crescimento, tais como intenções e aspirações de crescimento (DALBORG et al., 2012; BULANOVA et al., 2016; VENUGOPAL, 2016), estratégias de crescimento (MITCHELMORE; ROWLEY, 2013; REICHBORN-KJENNEREUD; SVARE, 2014) e a influência de networks sobre o crescimento (BOGREN et al., 2013). Contudo, determinantes e dificuldades de crescimento não foram investigados anteriormente, exceto Coad e Tamvada (2012), que exploraram apenas barreiras para crescimento de pequenos negócios em geral, porém sem foco em empreendedoras.

Estudos anteriores realizados com mulheres empreendedoras no Brasil focaram aspectos como desafios encontrados para gerir empresas (BOMFIM, TEIXEIRA, 2015) ou para criar empreendimentos (MACHADO et al., 2016), sem, contudo, focar no crescimento de empreendimentos criados e geridos por mulheres, sendo que é importante estudar o crescimento porque as empresas de mulheres representam aproximadamente $30 \%$ das pequenas empresas no país (SEBRAE, 2015).

Assim, esta pesquisa teve como objetivo identificar determinantes e dificuldades de crescimento de empresas de mulheres, buscando identificar a relação entre determinantes e dificuldades com características das empresas (idade da empresa e montante do 
capital inicial) e características das empreendedoras (idade que criou a empresa e nível de escolaridade). Inicialmente, apresenta-se uma revisão da literatura, focando em aspectos relativos ao crescimento de empresas de mulheres e, em seguida, abordam-se dificuldades e determinantes de crescimento para pequenos negócios.

\section{Crescimento de Empresas de Mulheres}

A pesquisa sobre crescimento de empresas, segundo Mckelvie e Wicklund (2010) pode ser classificada em três grupos, sendo: a) o crescimento como resultado (crescimento é variável dependente); b) o resultado do crescimento; c) o processo de crescimento. Quanto ao processo de crescimento, este requer abordagens que explorem o fenômeno além de variações quantitativas, que permitam identificar elementos como barreiras, dificuldades e intenções (DALBORG, 2015).

Há evidências de que o crescimento apresenta diferentes compreensões entre empreendedoras. Costin (2012) explorou compreensões de crescimento para mulheres empreendedoras e identificou que, para algumas, o crescimento representava aumento de lucratividade e vendas e, para outras, um equilíbrio entre trabalho e família. Para Dalborg et al. (2012), mais da metade das mulheres pesquisadas traduziram crescimento como sinônimo de sobrevivência, seguido de capacidade para criar empregos e desejo de obter estabilidade. Nesse estudo, o desenvolvimento pessoal foi outro significado apontado por um número reduzido de empreendedoras. É importante ressaltar que Dalborg et al. (2012) identificaram variações no significado de crescimento para empreendedoras em função do estágio de seus negócios: elas almejavam crescer apenas depois de atingir certa estabilidade nos negócios. No estágio inicial, a lucratividade e os ganhos para se manter nos negócios consistiram significados de crescimento. No estágio seguinte, a estabilidade era um sinônimo de crescimento e, no terceiro estágio, eles identificaram diferente visão do crescimento, sendo esta traduzida como desejo por boa reputação e apreço.

Naperspectivaprocessual,umdos aspectos pesquisados é o desejo de crescimento por empreendedoras. Há evidências de que empreendedoras preferem deixar suas empresas pequenas (REICHBORN-KJENNERUD; SVARE, 2014), para assegurar a qualidade dos produtos e serviços ofertados (BULANOVA et al.
2016). Ao mesmo tempo, Mitchelmore e Rowley (2013) identificaram que a melhoria de produtos ou serviços, assim como propaganda e promoção, foram estratégias escolhidas por empreendedoras para promover o crescimento dos negócios. Não há consenso sobre o desejo de crescimento, sendo que Bulanova et al. (2016) identificaram variações entre empreendedoras, e que algumas desejavam crescer por razões associadas ao negócio, à empreendedora e ao ambiente.

Quanto a intenções de crescimento por empreendedoras, Dalborg et al. (2012) identificaram intenções associadas a fatores extrínsecos, motivados por recompensas externas como dinheiro, por exemplo, e fatores intrínsecos, motivados por aspectos do comportamento, como satisfação. Venugopal (2016) identificou a influência da família na intenção de crescimento.

Esses estudos focaram em significados, intenções e estratégias de crescimento. No entanto, as intenções representam apenas uma parte de determinantes de crescimento, sendo importante identificar outras, a fim de ampliar a compreensão do processo de crescimento de empresas por mulheres.

\section{Determinantes de Crescimento}

Determinantes de crescimento são fatores que podem influenciar favoravelmente o crescimento. Segundo Wiklund et al. (2009) esses fatores podem ser associados ao indivíduo, à empresa ou ao ambiente.

Quanto aos fatores associados ao indivíduo, conforme comentado anteriormente, a intenção de crescer por parte de empreendedores pode influenciar o crescimento. Ademais, há evidências de que a escolha do tipo de negócio pode determinar o crescimento (MORRIS et al., 2006). Baron e Shane (2007) afirmam que empresas que iniciam em setores que estão crescendo têm mais chances de crescer. Mesmo assim, o conhecimento prévio do setor de atuação pelo empreendedor ou empreendedora pode contribuir para o crescimento do empreendimento (ROOMI et al., 2009). Empreendedores que abrem empresas em setores que conheciam previamente, ou em que tinham experiências, apresentam maiores chances de crescimento (BARON, SHANE, 2007). Essas chances podem ser aumentadas se o empreendedor tiver acesso a recursos financeiros, que tendem a 
influenciar positivamente o crescimento (ORHAN, 2001; MARLOW; PATTON, 2005; BRUIN ET AL., 2007).

Estudos anteriores apontaram ainda a influência do apoio da família (BOGREN ET AL., 2013; VENUGOPAL, 2016) para dar suporte à intensa dedicação e quantidade de horas de trabalho requeridas para promover o crescimento de empresas de mulheres (MARLOW; PATTON, 2005). Além das horas de dedicação requeridas para a gestão do negócio, outras ações como a participação em redes sociais e de negócios influenciam o crescimento (DALBORG ET AL. 2012). A inserção em redes sociais, bem como contatos pessoais e com outros empreendedores influencia a visão do negócio, a aprendizagem coletiva e o crescimento (BOGREN ET AL., 2013; CHABAUD; NGIOL, 2005; JULIEN, 2010; MACHADO; JESUS, 2010). Além disso, a participação de empreendedoras em Feiras e em Eventos pode influenciar o crescimento das empresas (GREENE et al., 2003).

Determinantes em nível das empresas foram apontadas em estudos anteriores, tais como a qualidade do produto (BULANOVA et al., 2016) e a adoção de novas tecnologias (BRUSH, 1997), ou ainda a diversificação de produtos e serviços por meio de lançamento de novos produtos ou serviços (COSTIN, 2012) e a capacidade criativa e inovadora (FREEL; ROBSON, 2004; ROOMI, HARRISON; BEAUMONT-KERRIDGE， 2009). Entre aspectos gerenciais, destacam-se também a ênfase na qualificação da equipe e na utilização de propaganda, publicidade e promoções (COSTIN, 2012; MITCHELMORE; ROWLEY, 2013).

O Quadro 1 resume as determinantes de crescimento comentadas anteriormente.

Quadro 1. Determinantes de Crescimento

\begin{tabular}{|l|l|}
\hline \multicolumn{1}{|c|}{$\begin{array}{c}\text { DETERMINANTES } \\
\text { DE CRESCIMENTO }\end{array}$} & \multicolumn{1}{|c|}{ AUTOR } \\
\hline $\begin{array}{l}\text { Dedicar muito tempo ao } \\
\text { negócio }\end{array}$ & Marlow; Patton (2005). \\
\hline $\begin{array}{l}\text { Ter acesso a recursos } \\
\text { financeiros }\end{array}$ & $\begin{array}{l}\text { Orhan (2001); Marlow; } \\
\text { Patton (2005); Bruin et al. } \\
\text { (2007). }\end{array}$ \\
\hline $\begin{array}{l}\text { Ter produtos e serviços de } \\
\text { qualidade }\end{array}$ & Bulanova et al., (2016). \\
\hline
\end{tabular}

\begin{tabular}{|l|l|}
\hline $\begin{array}{l}\text { Ter uma equipe } \\
\text { qualificada }\end{array}$ & Costin (2012). \\
\hline $\begin{array}{l}\text { Ter conhecimento sobre o } \\
\text { negócio desde o início }\end{array}$ & Roomi et al. (2009). \\
\hline Ser criativo e inovador & Freel; Robson (2004). \\
\hline $\begin{array}{l}\text { Diversificar com novos } \\
\text { produtos e serviços }\end{array}$ & $\begin{array}{l}\text { Freel; Robson (2004); } \\
\text { Roomi et al.(2009); Costin } \\
\text { (2012) }\end{array}$ \\
\hline $\begin{array}{l}\text { Uso do marketing - } \\
\text { propaganda, promoções, } \\
\text { publicidade. }\end{array}$ & $\begin{array}{l}\text { Costin (2012); } \\
\text { Mitchelmore; Rowley } \\
\text { (2013). }\end{array}$ \\
\hline Adotar novas tecnologias & Brush (1997) \\
\hline $\begin{array}{l}\text { Participar de Feiras e } \\
\text { Eventos }\end{array}$ & Greene et al. (2003) \\
\hline $\begin{array}{l}\text { Participação em Redes } \\
\text { Sociais }\end{array}$ & $\begin{array}{l}\text { Chabaud; Ngiol (2005); } \\
\text { Dalborg et al. (2012); Julien } \\
\text { (2010); Machado; Jesus } \\
\text { (2010). }\end{array}$ \\
\hline Receber apoio da família & $\begin{array}{l}\text { Bogren et al. (2013); } \\
\text { Venugopal (2016) }\end{array}$ \\
\hline
\end{tabular}

\section{Dificuldades para 0 crescimento de empresas}

Segundo Storey (1994, p. 155), dificuldades de crescimento são "fatores internos e externos, ou as condições que limitam o potencial de crescimento de uma empresa que deseja crescer". Elas têm sido usadas como uma característica que permite fazer prognósticos para saber se as empresas são mais propensas a crescer ou não (ORSER et al., 2000).

Entre os aspectos que dificultam o crescimento de empresas e que podem ser associados ao nível do empreendedor ou da empreendedora, foram mencionados em estudos anteriores: pouca qualificação pessoal do empreendedor, reduzida formação gerencial e pouca experiência no segmento de atuação (DOLINSKY; CAPUTO, 2003; ROOMI et al., 2009), bem como o reduzido nível de autoconfiança (ROOMI et al., 2009; WINN, 2005) e a dificuldade em conciliar trabalho e família (SHELTON, 2006; GREENE et al., 2003; GANESAN et al., 2002). Além disso, o medo de empreendedores de assumir riscos pode dificultar o crescimento do empreendimento (HERMANS et al., 2012).

No entanto, não apenas dificuldades associadas ao indivíduo podem dificultar o crescimento do empreendimento, como também variáveis associadas 
ao meio, como, por exemplo, a escassez de recursos financeiros (MARTINS et al., 2010). Nesse sentido, Martins et al. (2010) afirmam que empreendedoras têm pouco acesso a recursos financeiros de terceiros, o que ocorre muitas vezes por restrições na concessão de crédito a mulheres. Há também evidências de que mulheres encontram dificuldades para participar em redes sociais e para construir relacionamentos comerciais, em função do pouco tempo livre para essas atividades (GUNDRY;WELSCH, 2001; GANESAN et al., 2002; SHELTON, 2006).

Ademais, para Roomi et al. (2009) mulheres enfrentam dificuldades para crescer pela reduzida capacidade de inovação, pela falta de informações sobre o negócio e pela falta de recursos tecnológicos e de equipamentos. Esses aspectos são relacionados à gestão do negócio, sendo que há outros, tais como falta de estratégias de crescimento e de funcionários qualificados, bem como falta de organização e de planejamento (BOMFIM; TEIXEIRA, 2015; DAVIDSSON et al., 2010; ROOMI et al., 2009). O Quadro 2 resume dificuldades para crescimento de empresas.

Quadro 2 . Dificuldades para crescimento de empresas

\begin{tabular}{|l|l|}
\hline \multicolumn{1}{|c|}{$\begin{array}{c}\text { Dificuldades para o } \\
\text { crescimento }\end{array}$} & \multicolumn{1}{|c|}{ Autores } \\
\hline $\begin{array}{l}\text { Falta de qualificação } \\
\text { pessoal }\end{array}$ & $\begin{array}{l}\text { Dolinsky; Caputo (2003); } \\
\text { Brush (1992); Roomi } \text { et al. } \\
\text { (2009); Winn (2005). }\end{array}$ \\
\hline Falta de autoconfiança & $\begin{array}{l}\text { Roomi } \text { et al. (2009); Winn } \\
\text { (2005) }\end{array}$ \\
\hline Não ser inovadora & Roomi et. al. (2009) \\
\hline $\begin{array}{l}\text { Falta de planejamento da } \\
\text { empresa }\end{array}$ & Davidsson et al. (2010) \\
\hline $\begin{array}{l}\text { Falta de organização da } \\
\text { empresa }\end{array}$ & Davidsson et al. (2010) \\
\hline $\begin{array}{l}\text { Falta de informações } \\
\text { sobre o negócio }\end{array}$ & Roomi et al. (2009) \\
\hline $\begin{array}{l}\text { Falta de estratégia de } \\
\text { crescimento }\end{array}$ & Davidsson et al. (2010) \\
\hline $\begin{array}{l}\text { Não ter facilidade para } \\
\text { construir relacionamentos } \\
\text { comerciais }\end{array}$ & $\begin{array}{l}\text { Ganesan et al. (2002); } \\
\text { Gundry; Welsch (2001). }\end{array}$ \\
\hline $\begin{array}{l}\text { Não participar de redes } \\
\text { de negócios }\end{array}$ & $\begin{array}{l}\text { Roomi et al. (2009); } \\
\text { Gundry; Welsch (2001). }\end{array}$ \\
\hline $\begin{array}{l}\text { Não participar de Feiras e } \\
\text { Eventos }\end{array}$ & Greene et al. (2003) \\
\hline
\end{tabular}

\begin{tabular}{|l|l|}
\hline Falta de equipamentos & Roomi et al. (2009) \\
\hline $\begin{array}{l}\text { Falta de recursos } \\
\text { tecnológicos }\end{array}$ & Roomi et al. (2009) \\
\hline $\begin{array}{l}\text { Falta de empregados } \\
\text { qualificados }\end{array}$ & Davidsson et al (2010) \\
\hline $\begin{array}{l}\text { Não ter acesso a recursos } \\
\text { financeiros }\end{array}$ & Martins et al. (2010). \\
\hline $\begin{array}{l}\text { Não ter facilidade para } \\
\text { assumir riscos }\end{array}$ & Hermans et al. (2012) \\
\hline $\begin{array}{l}\text { Não conseguir conciliar } \\
\text { trabalho e família }\end{array}$ & $\begin{array}{l}\text { Shelton. (2006); Greene et } \\
\text { al. (2003); Ganesan et al. } \\
\text { (2002). }\end{array}$ \\
\hline
\end{tabular}

Considerando as determinantes e dificuldades de crescimento, apresentadas nos Quadro 1 e 2, respectivamente, esta pesquisa apresenta uma abordagem processual do crescimento de empresas de mulheres, que pode ser visualizada na Figura 1, apresentando o modelo teórico da pesquisa. Como variáveis moderadoras, foram consideradas características da empreendedora: idade quando abriram as empresas e nível de escolaridade e características do empreendimento: idade e capital inicial.

Figura 1. Modelo Teórico: Determinantes e Dificuldades de Crescimento de Empresas de Mulheres

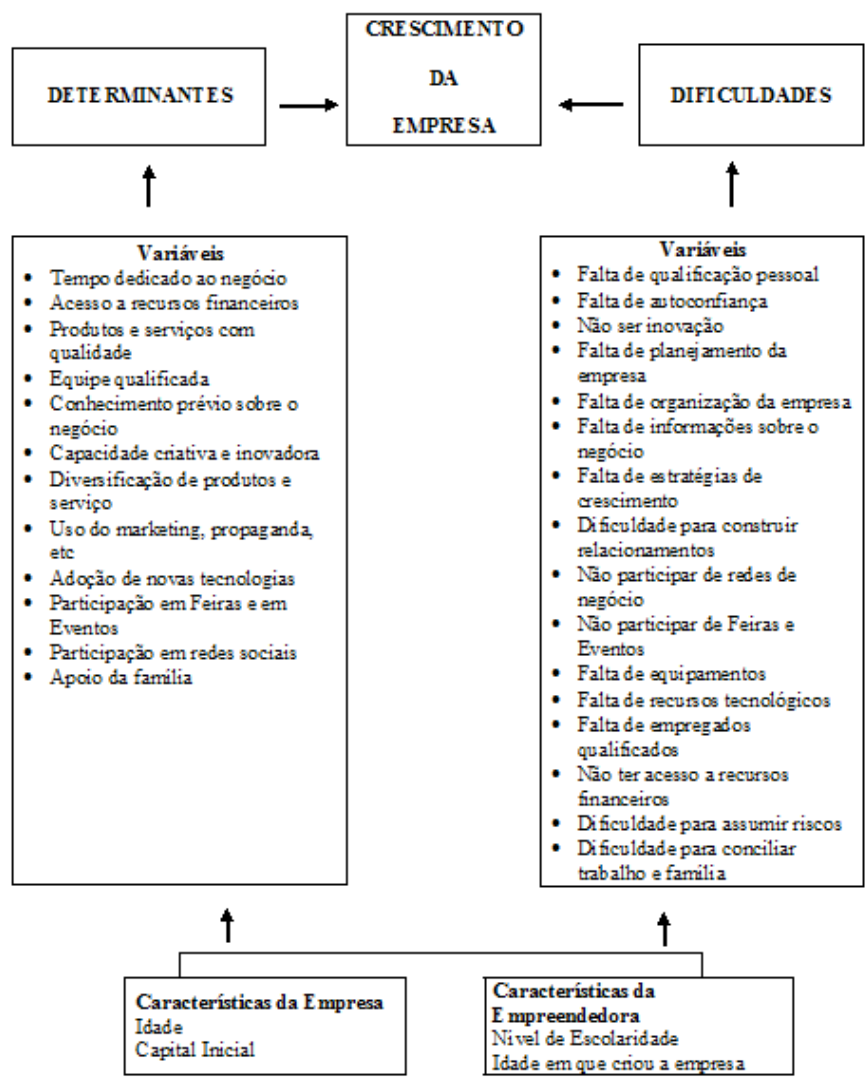




\section{Procedimentos Metodológicos}

Esta pesquisa é um estudo exploratório, no que se refere à identificação de determinantes e dificuldades de crescimento para empreendedoras e descritivo, ao buscar relacionar essas variáveis com características da empresa e das empreendedoras.

O contexto da pesquisa foi o setor de confecções, sendo um dos setores que conta com várias empresas de mulheres. A pesquisa foi realizada no Estado do Paraná, sendo que, neste local, o setor de confecções é o segundo polo industrial do país, com uma produção de 150 milhões de peças, conforme dados do Sindicato da Indústria do Vestuário (SINDVEST, 2009).

A amostra foi constituída por 182 empreendedoras do setor de confecção, filiadas ao Sindicato da Indústria do Vestuário, que eram proprietárias de pelo menos 50\% do capital da empresa. Dos 182 questionários enviados, 102 retornaram.

A coleta de dados foi realizada por meio de questionário estruturado, constituído por questões fechadas. O primeiro bloco de questões versava sobre dados da caracterização das empreendedoras (idade no momento da criação da empresa, nível de escolaridade e estado civil) e das empresas (ano de criação e valor do capital inicial). Além disso, duas questões foram estruturadas no formato de escala do tipo Likert, cujas medidas variavam de 1 a 5 , sendo 1 representando "discordo totalmente" e 5 "concordo totalmente", referente a determinantes e dificuldades de crescimento. As variáveis apresentadas nos Quadros 1 e 2 foram transferidas para as escalas utilizadas, de forma a mensurar as dificuldades e as determinantes do crescimento para as empreendedoras que participaram da pesquisa. Foram enviados questionários a todas as empreendedoras, sendo o percentual de retorno de $56,04 \%$.

Para análise dos dados, foram realizadas análises descritivas e exploratórias. A média foi utilizada para identificar, entre uma coleção de atributos, os mais expressivos. O teste de Spearman foi utilizado para investigar a correlação entre determinantes e dificuldades de crescimento (HAIR et al., 2009) com as características das empresas e das empreendedoras, sendo ano de criação e capital inicial, e idade ao criar a empresa e nível de escolaridade, respectivamente.

Além dessas análises, a de Cluster foi realizada com a finalidade de identificar grupos de atributos similares relacionados às determinantes e às dificuldades de crescimento das empresas. A medida utilizada foi distância euclidiana, uma medida de similaridade frequentemente utilizada (REIS, 1997). O critério de agregação (ou desagregação) das variáveis distinguiuse pela forma como as distâncias foram estimadas entre grupos formados e outros grupos por agrupar, tendo sido empregado o critério de agregação single linkage ou critério do vizinho mais próximo, que se baseia na distância mínima. A distância entre dois grupos de variáveis foi determinada pela distância entre dois elementos mais próximos em diferentes grupos.

\section{Apresentação e Análises dos Dados}

Com relação às características das empresas, estas foram abertas entre os anos de 1977 e 2009, como mostra a Tabela 1. A maioria das empresas entrou no mercado depois de 1996 e a empresa mais antiga foi criada em 1977.

Tabela 1 . Ano de criação das empresas

\begin{tabular}{ccc}
\hline Época da criação & Freqüência & Percentual \\
\hline 1977 a 1988 & 11 & 10,8 \\
1989 a 1995 & 20 & 19,6 \\
1996 a 2002 & 27 & 26,5 \\
2003 a 2009 & 44 & 43,1 \\
\hline TOTAL & 102 & 100,00 \\
\hline
\end{tabular}

A distribuição do capital inicial pode ser visualizada na Tabela 2. Aproximadamente a metade das empresas $(49,1 \%)$ foi aberta com capital igual ou inferior a $\mathrm{R} \$$ $20.000,00$. No entanto, a variação entre o menor e o maior capital social é de 200 vezes, sendo o menor capital inicial $\mathrm{R} \$ 2.000,00$ e o maior $\mathrm{R} \$ 400.000,00$.

Tabela 2 . Montante do capital inicial das empresas

\begin{tabular}{lcc}
\hline \multicolumn{1}{c}{ Capital Inicial } & Freqüência & Percentual \\
\hline Até R \$ 10.000,00 & 33 & 32,4 \\
Entre R $\$ 10.001,00$ e & 17 & 16,7 \\
R \$ 20.000,00 & & \\
Entre R $\$ 20.001,00$ e & 7 & 6,9 \\
R $\$ 30.000,00$ & &
\end{tabular}




\begin{tabular}{lcc} 
Entre $\mathrm{R} \$ 30.001,00$ e & 6 & 5,9 \\
$\mathrm{R} \$ 40.000,00$ & & \\
Acima de R $\$ 40.000,00$ & 17 & 16,7 \\
Não informaram & 22 & 21,6 \\
\hline \multicolumn{1}{c}{ TOTAL } & 102 & 100,00 \\
\hline
\end{tabular}

Com relação ao perfil das empreendedoras, as Tabelas 3 e 4 mostram, respectivamente, o nível de escolaridade e a idade que as empreendedoras tinham quando criaram as empresas. O nível de escolaridade predominante é o segundo grau, seguido de superior completo.

Tabela 3 . Nível de escolaridade das empreendedoras

\begin{tabular}{lcc}
\hline \multicolumn{1}{c}{ Escolaridade } & Freqüência & Percentual \\
\hline Primeiro Grau & 17 & 16,7 \\
Segundo Grau & 50 & 49,0 \\
Superior & 31 & 30,4 \\
Pós graduação & 4 & 3,9 \\
\hline \multicolumn{1}{c}{ TOTAL } & 102 & 100,00 \\
\hline
\end{tabular}

A maioria das empresas $(52 \%)$ foi criada quando as empreendedoras tinham menos de 30 anos de idade e apenas $24 \%$ foram criadas quando elas tinham idade superior a 35 anos.

Tabela 4 . Idade quando criaram as empresas

\begin{tabular}{lcc}
\hline \multicolumn{1}{c}{ Idade } & Freqüência & Percentual \\
\hline Menos que 20 anos & 4 & 3,9 \\
De 20 a 24 anos & 22 & 21,6 \\
De 25 a 29 anos & 26 & 25,5 \\
De 30 a 34 anos & 26 & 25,5 \\
De 35 a 39 anos & 12 & 11,8 \\
Mais que 40 anos & 12 & 11,8 \\
\hline \multicolumn{1}{c}{ TOTAL } & 102 & 100,00 \\
\hline
\end{tabular}

Em síntese, as empreendedoras iniciaram as empresas com reduzidos capitais iniciais, sendo que a maioria das empresas foi aberta com capital inicial inferior a $\mathrm{R} \$ 30.000,00$. A empresa mais velha, no momento da pesquisa, tinha 34 anos e a mais nova tinha três anos. O nível de escolaridade para 35\% dos casos é superior completo ou pós graduação e as empresas foram criadas, na maioria dos casos, quando elas tinham entre 20 e 34 anos de idade.

\section{Determinantes para o Crescimento}

As determinantes para o crescimento das empresas constam na Tabela 5. As determinantes: o tempo dedicado ao negócio, o conhecimento prévio da atividade, a capacidade de ser criativa e inovadora e a diversificação de produtos e serviços foram consideradas as mais importantes. A análise de Cluster (Figura 2) mostra o agrupamento das determinantes $\mathrm{S} 1, \mathrm{~S} 5, \mathrm{~S} 6$ e S7, corroborando com os resultados da Tabela 5.

Para as empreendedoras, entre as principais determinantes do crescimento consta o conhecimento prévio do negócio, corroborando resultados de Rommi et al. (2009). Entretanto, convém salientar que o conhecimento prévio do negócio é apontado na literatura como uma condição importante para a criação da empresa (SHANE; VENKATARAMAN, 2000), e não para promover o crescimento do negócio, como mostrou esta pesquisa.

Outra determinante considerada importante para o crescimento pelas empreendedoras desta pesquisa foi o tempo dedicado ao negócio, sendo que, para promover o crescimento das empresas, é necessário dedicar muito tempo ao negócio. Este resultado foi também encontrado por Marlow e Patton (2005) anteriormente. Além destas determinantes comentadas, também a capacidade de ser criativa e inovadora e a diversificação de produtos e serviços foram consideradas por elas como importantes para o crescimento do negócio. É possível que estas últimas determinantes sejam influenciadas pelo setor de atividades que elas atuam, que é o de confecções.

Tabela 5. Determinantes para o Crescimento de Empresas

\begin{tabular}{llll}
\hline Ordem & Determinantes & Média & $\begin{array}{c}\text { Desvio } \\
\text { padrão }\end{array}$ \\
\hline S1 & $\begin{array}{l}\text { Dedicar muito } \\
\text { tempo ao negócio }\end{array}$ & $\mathbf{4 , 3 1 4}$ & 0,808 \\
S2 & $\begin{array}{l}\text { Ter acesso e } \\
\text { controle dos } \\
\text { recursos financeiros }\end{array}$ & 3,971 & 1,156 \\
& & &
\end{tabular}




\begin{tabular}{|c|c|c|c|}
\hline S3 & $\begin{array}{l}\text { Ter produtos } \\
\text { e serviços de } \\
\text { qualidade }\end{array}$ & 3,108 & 1,281 \\
\hline S4 & $\begin{array}{l}\text { Ter uma equipe } \\
\text { qualificada }\end{array}$ & 2,461 & 1,132 \\
\hline S5 & $\begin{array}{l}\text { Ter conhecimento } \\
\text { sobre o negócio } \\
\text { desde o início }\end{array}$ & 4,441 & 0,791 \\
\hline S6 & $\begin{array}{l}\text { Ser criativa e } \\
\text { inovadora }\end{array}$ & 4,627 & 0,644 \\
\hline S7 & $\begin{array}{l}\text { Diversificar com } \\
\text { novos produtos e } \\
\text { serviços }\end{array}$ & 4,167 & 1,135 \\
\hline S8 & $\begin{array}{l}\text { Uso do marketing } \\
\text { - propaganda, } \\
\text { promoções, } \\
\text { publicidade, etc. }\end{array}$ & 2,412 & 1,230 \\
\hline S9 & $\begin{array}{l}\text { Adotar novas } \\
\text { tecnologias }\end{array}$ & 2,696 & 1,326 \\
\hline S10 & $\begin{array}{l}\text { Participar de Feiras } \\
\text { e Eventos }\end{array}$ & 3,451 & 1,279 \\
\hline S11 & Fazer parte de redes & 2,853 & 1,360 \\
\hline S12 & $\begin{array}{l}\text { Receber apoio da } \\
\text { família }\end{array}$ & 2,980 & 1,364 \\
\hline
\end{tabular}

As determinantes menos importantes, de acordo com os resultados mostrados na Tabela 5, foram o uso do marketing- propaganda, promoções, publicidade e a condição de ter uma equipe qualificada.

Figura 2. Análise de Cluster para Determinantes de crescimento para as empreendedoras

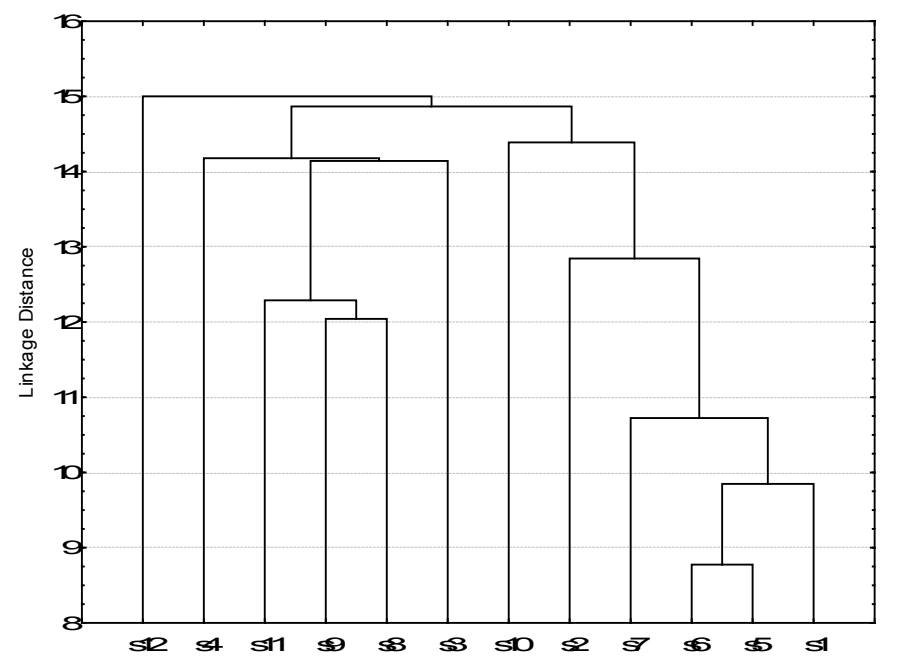

A análise de cluster, mostrada na figura 2, reforça os resultados encontrados por meio do cálculo das médias, uma vez que agrupa as determinantes dedicar muito tempo ao negócio, ter conhecimento sobre o negócio desde o início, ser criativa e inovadora e diversificar com novos produtos e serviços, evidenciando que estas determinantes de crescimento foram similares para um grupo de empreendedoras.

\section{Dificuldades para 0 Crescimento}

Quanto às dificuldades para o crescimento das empresas, como mostram os dados na Tabela 6, três delas estão relacionadas com características das empreendedoras: falta de qualificação pessoal, falta de autoconfiança e perfil não inovador. Especificamente falta de autoconfiança e de qualificação pessoal foram dificuldades identificadas em estudos anteriores (SHELTON, 2006; GREENE et al., 2003).

Outro grupo, representado por quatro dificuldades, está relacionado à gestão das empresas: falta de planejamento, falta de organização, falta de informações e falta de estratégias de crescimento. Quanto às estratégias de crescimento para empreendedoras, o estudo de Mitchelmore e Rowley (2013) identificou que o horizonte de planejamento para elas é de curto prazo, resultando em poucas estratégias de crescimento.

Por outro lado, variáveis relacionadas ao ambiente externo, como, por exemplo, a dificuldade em obter recursos financeiros, não constou entre as principais dificuldades de crescimento para as empreendedoras desta pesquisa. Resultado diferente foi apontado por Fleck et al. (2011), analisando um grupo de 33 empreendedoras na Irlanda que mencionaram a dificuldade em obter recursos financeiros como limitação para crescimento. No entanto, elas também mencionaram a falta de autoconfiança, que foi uma das principais dificuldades para as mulheres nesta pesquisa.

A figura 3 apresenta os resultados da análise de cluster, evidenciando que as principais dificuldades apontadas na Tabela 6 se agrupam (D1 a D7). 
Tabela 6 . Dificuldades para o crescimento

\begin{tabular}{|c|c|c|c|}
\hline Ordem & Dificuldades & Média & $\begin{array}{l}\text { Desvio } \\
\text { padrão }\end{array}$ \\
\hline D1 & $\begin{array}{l}\text { Falta de } \\
\text { qualificação pessoal }\end{array}$ & 4,294 & 1,086 \\
\hline D2 & $\begin{array}{l}\text { Falta de } \\
\text { autoconfiança }\end{array}$ & 4,284 & 1,172 \\
\hline D3 & Não ser inovadora & 4,245 & 1,164 \\
\hline D4 & $\begin{array}{l}\text { Falta de } \\
\text { planejamento da } \\
\text { empresa }\end{array}$ & 4,304 & 1,106 \\
\hline D5 & $\begin{array}{l}\text { Falta de } \\
\text { organização da } \\
\text { empresa }\end{array}$ & 4,324 & 1,101 \\
\hline D6 & $\begin{array}{l}\text { Falta de } \\
\text { informações sobre } \\
\text { o negócio }\end{array}$ & 4,333 & 1,075 \\
\hline D7 & $\begin{array}{l}\text { Falta de estratégia } \\
\text { de crescimento }\end{array}$ & 4,176 & 1,066 \\
\hline D8 & $\begin{array}{l}\text { Não ter facilidade } \\
\text { para construir } \\
\text { relacionamentos } \\
\text { comerciais }\end{array}$ & 4,088 & 1,187 \\
\hline D9 & $\begin{array}{l}\text { Não participar de } \\
\text { redes de negócio }\end{array}$ & 3,176 & 1,438 \\
\hline D10 & $\begin{array}{l}\text { Não participar de } \\
\text { feiras e eventos }\end{array}$ & 3,480 & 1,333 \\
\hline D11 & $\begin{array}{l}\text { Falta de } \\
\text { equipamentos }\end{array}$ & 3,627 & 1,364 \\
\hline D12 & $\begin{array}{l}\text { Falta de recursos } \\
\text { tecnológicos }\end{array}$ & 3,549 & 1,354 \\
\hline D13 & $\begin{array}{l}\text { Falta de } \\
\text { empregados } \\
\text { qualificados }\end{array}$ & 4,137 & 1,235 \\
\hline D14 & $\begin{array}{l}\text { Não ter acesso a } \\
\text { recursos financeiros }\end{array}$ & 3,863 & 1,178 \\
\hline D15 & $\begin{array}{l}\text { Não ter facilidade } \\
\text { para assumir riscos }\end{array}$ & 3,892 & 1,177 \\
\hline D16 & $\begin{array}{l}\text { Não conseguir } \\
\text { conciliar trabalho e } \\
\text { família }\end{array}$ & 4,078 & 1,240 \\
\hline
\end{tabular}

Figura 3. Análise de Cluster para dificuldades de crescimento de empresas para empreendedoras

A análise de cluster, apresentada na figura 3, mostra o agrupamento quanto às dificuldades para o crescimento, reiterando os resultados apresentados na Tabela 6, incluindo dificuldades pessoais (falta

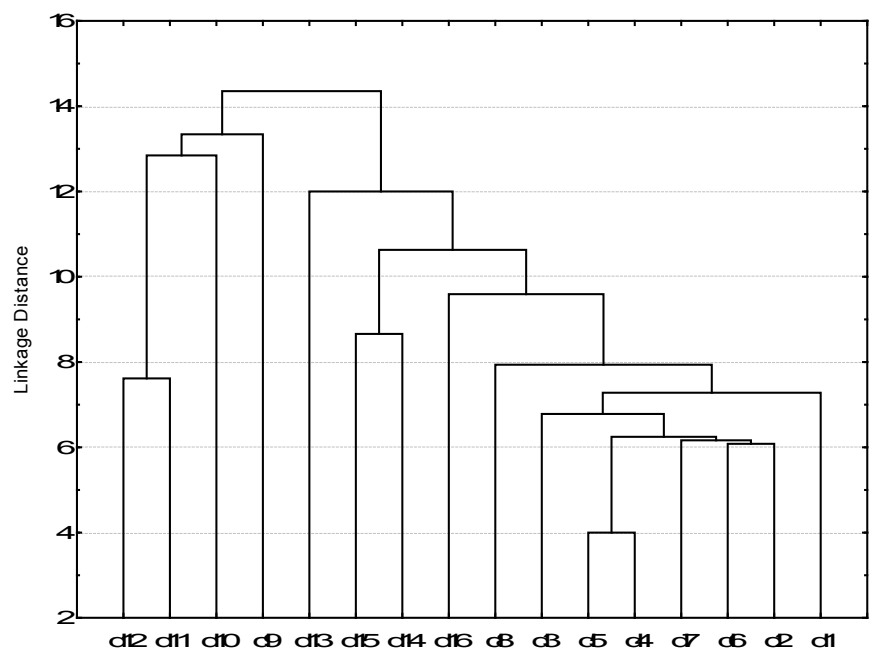

de qualificação pessoal, falta de autoconfiança e perfil não inovador) e dificuldades gerenciais (falta de planejamento, falta de organização, falta de informações e falta de estratégias de crescimento).

\section{Correlação entre Dificuldades e Determinantes para 0 crescimento com características das empresas e das empreendedoras}

As correlações entre determinantes de crescimento e características das empreendedoras: nível de escolaridade e idade quando criaram as empresas, bem como entre determinantes de crescimento e características das empresas: idade e capital inicial são mostradas na Tabela 7.

A dificuldade menos relevantes para o crescimento foi a não participação em redes de negócios, no entanto foi a variável que apresentou o maior desvio padrão $(1,438)$. 
Tabela 7 . Coeficiente de correlação entre determinantes e escolaridade e idade da empreendedora e idade e capital inicial da empresa.

\begin{tabular}{lcccc}
\hline \multicolumn{1}{c}{$\begin{array}{c}\text { Determinantes de } \\
\text { Crescimento }\end{array}$} & $\begin{array}{c}\text { Escolaridade da } \\
\text { empreendedora }\end{array}$ & $\begin{array}{c}\text { Idade que a } \\
\text { empreendedora } \\
\text { criou a empresa }\end{array}$ & $\begin{array}{c}\text { Idade da } \\
\text { Empresa }\end{array}$ & Capital Inicial \\
\hline $\begin{array}{l}\text { Dedicar muito tempo ao } \\
\text { negócio }\end{array}$ & $-0,142$ & 0,040 & $-0,081$ & 0,091 \\
$\begin{array}{l}\text { Ter acesso e controle dos } \\
\text { recursos financeiros }\end{array}$ & $-0,138$ & $-0,063$ & $-0,018$ & $-0,014$ \\
$\begin{array}{l}\text { Ter produtos e serviços de } \\
\text { qualidade }\end{array}$ & $-0,024$ & $-0,148$ & $-0,012$ & $-0,062$ \\
$\begin{array}{l}\text { Ter uma equipe qualificada } \\
\text { Ter conhecimento sobre o }\end{array}$ & 0,136 & $-0,180$ & 0,005 & 0,049 \\
negócio desde o início & $-0,126$ & $-0,143$ & $-0,029$ & $-0,164$ \\
Ser criativo e inovador & 0,084 & 0,001 & $-0,138$ & $-0,041$ \\
$\begin{array}{l}\text { Diversificação com novos } \\
\text { produtos e serviços }\end{array}$ & $-0,110$ & $* 0,042$ & $*-0,254$ & $-0,008$ \\
Adotar novas tecnologias & 0,094 & 0,020 & $-0,080$ & $-0,098$ \\
Uso do marketing - & 0,094 & $-0,091$ & $-0,198$ & 0,013 \\
$\begin{array}{l}\text { propaganda, promoções, } \\
\text { publicidade, etc. }\end{array}$ & & & & $-0,241$ \\
$\begin{array}{l}\text { Participar de Feiras e Eventos } \\
\text { Participar de Redes }\end{array}$ & $-0,070$ & $-0,017$ & $-0,091$ & $-0,033$ \\
Receber apoio da família & 0,069 & $-0,048$ & $-0,024$ & $-0,139$ \\
\hline
\end{tabular}

Nota. O símbolo * indica que a variável é significativa ao nível 5\%.

Duas determinantes de crescimento apresentaram correlação com a idade quando a empreendedora criou a empresa. A primeira foi com a diversificação de produtos e serviços, ou seja, quanto maior a idade que tinham ao criarem as empresas, maior a importância atribuída à diversificação com novos produtos e serviços para o crescimento. A segunda correlação foi com a participação em Feiras e eventos, mas esta foi negativa, sendo que quanto mais jovens eram quando criaram as empresas, maior importância foi atribuída à participação em Feiras e eventos para o crescimento dos negócios. O nível de escolaridade da empreendedora apresentou correlação negativa com o apoio recebido da família.

Quanto às características da empresa, a diversificação de produtos e serviços apresentou correlação com a idade da empresa, sendo que as mais jovens consideraram menos determinante a diversificação com novos produtos e serviços para o crescimento. Isso pode ser explicado pelo fato das empresas mais jovens necessitarem primeiramente consolidar a marca no mercado para depois pensar em diversificação do produto.

Por sua vez, o montante do capital inicial não apresentou correlação com determinantes de crescimento. Mesmo com variações encontradas no capital inicial, como pode ser visualizado na Tabela 2, não há correlação com determinantes de crescimento.

A correlação entre dificuldades de crescimento e características das empreendedoras (idade quando criaram as empresas e nível de escolaridade) e entre dificuldades de crescimento e características das empresas (idade e montante do capital inicial) é mostrada na Tabela 8. 
Tabela 8 . Coeficiente de correlação entre dificuldades de crescimento, nível de escolaridade e idade da empreendedora, idade e capital inicial da empresa.

\begin{tabular}{lcrrr}
\hline \multicolumn{1}{c}{ Dificuldades para Crescimento } & $\begin{array}{c}\text { Escolaridade da } \\
\text { empreendedora }\end{array}$ & $\begin{array}{c}\text { Idade que a } \\
\text { empreendedora criou a } \\
\text { empresa }\end{array}$ & $\begin{array}{c}\text { Idade da } \\
\text { Empresa }\end{array}$ & $\begin{array}{c}\text { Capital } \\
\text { Inicial }\end{array}$ \\
\hline Falta de qualificação pessoal & $-0,008$ & 0,143 & 0,033 & $-0,101$ \\
Falta de autoconfiança & $-0,157$ & 0,042 & 0,032 & $-0,056$ \\
Não ser inovadora & $-0,182$ & 0,013 & $-0,007$ & $-0,188$ \\
Falta de planejamento da empresa & $*-0,277$ & $-0,083$ & 0,019 & $-0,139$ \\
Falta de organização da empresa & $*-0,272$ & $-0,083$ & 0,077 & $-0,219$ \\
Falta de informações sobre o negócio & $* 0,220$ & 0,054 & 0,028 & $-0,089$ \\
Falta de estratégias de crescimento & $-0,120$ & $-0,009$ & $-0,017$ & $-0,052$ \\
Não ter facilidade para construir & $-0,185$ & 0,007 & 0,069 & 0,056 \\
relacionamentos comerciais & $-0,004$ & 0,149 & $*-0,204$ & $-0,063$ \\
Não participar de Redes de negócios & $-0,151$ & 0,065 & 0,1103 & $-0,044$ \\
Não participar de Feiras e Eventos & $*-0,311$ & 0,001 & $-0,005$ & $-0,111$ \\
Falta de equipamentos & $*-0,306$ & $-0,076$ & $-0,047$ & $-0,140$ \\
Falta de recursos tecnológicos & 0,031 & 0,0170 & 0,051 & $-0,147$ \\
Falta de empregados qualificados & $-0,128$ & $-0,064$ & 0,005 & 0,034 \\
Não ter facilidade para assumir riscos & $-0,144$ & 0,101 & 0,006 & $-0,077$ \\
Não ter acesso a recursos financeiros & $*-0,206$ & 0,006 & $-0,009$ & $-0,022$ \\
Não conseguir conciliar trabalho e & & & & \\
família & & &
\end{tabular}

Nota. O símbolo * indica que a variável é significativa nível 5\%.

Observa-se na Tabela 8 que o nível de escolaridade das empreendedoras apresentou correlação negativa com as seguintes dificuldades: falta de planejamento e de organização, dificuldade em conciliar trabalho e família, falta de equipamentos e falta de recursos tecnológicos, sendo que, quanto menor o nível de escolaridade, mais essas constituem dificuldades para o crescimento. Além disso, a falta de informações sobre o negócio apresentou correlação positiva com o nível de escolaridade, sendo que, quanto maior o nível de escolaridade da empreendedora, maior a importância de superar essa dificuldade para alcançar o crescimento do negócio. A idade que elas tinham quando criaram as empresas não apresentou correlação com as dificuldades para crescimento.

Quanto às características da empresa, apenas a idade apresentou correlação negativa com dificuldade de não participar em redes, sendo que quanto menor a idade da empresa, mais importante é a dificuldade de participar em redes de negócios. Isso pode ser explicado pela necessidade de se promover a consolidação da empresa, o que requer intensa dedicação e quantidade de horas de trabalho (MARLOW; PATTON, 2005) e, deste modo, não sobra tempo para outras atividades, como a participação em redes, podendo então limitar o crescimento dos negócios (GANESAN et al., 2002; SHELTON, 2006). Nesta pesquisa foi identificada correlação com dificuldades de participação em redes e Dalborg (2015) identificaram correlação entre a idade da empresa e aspirações de crescimento por empreendedoras.

Por fim, nesta pesquisa não foi identificada correlação entre dificuldades de crescimento e montante do capital inicial, mesmo com a variação no capital inicial. As dificuldades para crescimento não estão relacionadas com a idade que as empreendedoras criaram as empresas ou com a quantidade de recursos financeiros aplicadas no empreendimento. 


\section{Considerações Finais}

O objetivo desta pesquisa foi identificar determinantes e dificuldades de crescimento de empresas de mulheres, assim como investigar a relação entre estas variáveis e características das empresas e das empreendedoras. É importante salientar que a pesquisa apresentou uma contribuição sobre determinantes e dificuldades para o crescimento de empresas por mulheres em um setor que conta tradicionalmente com a participação de mulheres, que é o de confecções.

Para as empreendedoras desta pesquisa o que determina o crescimento da empresa é quantidade de horas de trabalho dedicadas ao negócio, o conhecimento prévio, a capacidade de ser criativa e inovadora e a diversificação de produtos e serviços. Entre estas determinantes apenas a diversificação de produtos e serviços apresentou correlação positiva com a idade que a empreendedora tinha quanto criou a empresa e correlação negativa com a idade da empresa. As determinantes: horas dedicadas ao trabalho, capacidade de ser criativa e inovadora e conhecimento prévio foram relevantes independente da idade da empresa e da idade com a qual as empreendedoras criaram as empresas, ressaltando que $52 \%$ das empresas foram criadas quando elas tinham menos de 30 anos de idade. Outras correlações entre determinantes de crescimento com características das empreendedoras foram observadas na pesquisa: participação em Feiras e Eventos, que apresentou correlação negativa com a idade no momento da criação da empresa e receber o apoio da família apresentou correlação negativa com o nível de escolaridade das empreendedoras.

De acordo com Wiklund et al. (2009) o crescimento está associado a variáveis do ambiente, da empresa e do indivíduo. As empreendedoras nesta pesquisa apontaram principalmente variáveis associadas ao indivíduo, sendo somente a diversificação do produto e de serviços associada à empresa e nenhuma variável do ambiente foi mencionada como determinante do crescimento. A importância do apoio da família para o crescimento, destacada por Venugopal (2016) não aparece entre as determinantes que se agruparam nesta pesquisa.

Sobre as principais dificuldades para o crescimento estas foram associadas ao perfil pessoal, tais como baixa capacidade inovadora, falta de qualificação profissional e falta de autoconfiança. Convém salientar que a autoconfiança é um atributo importante para o crescimento dos negócios (FLECK et al., 2011;
MORRIS et al., 2006), e foi também constatada por Fleck et al. (2011) entre empreendedoras na Irlanda. Esta dificuldade pode ser uma das razões que levam os negócios de mulheres a permanecerem pequenos, como salientam Morris et al. (2006).

Além das dificuldades associadas ao perfil pessoal, foram identificadas outras associadas ao perfil gerencial, como: falta de organização e de planejamento, falta de informações sobre o negócio e falta de estratégias de crescimento. As dificuldades de crescimento não apresentaram correlação com características da empresa, ou seja, com o montante do capital inicial e também não apresentaram correlação com a característica da empreendedora, a idade com a qual criou a empresa. No entanto, o nível de escolaridade das empreendedoras apresentou correlação positiva com a falta de informações sobre o negócio. Correlação negativa foi observada entre o nível de escolaridade da empreendedora e as dificuldades: falta de planejamento e de organização da empresa, falta de equipamentos e de recursos tecnológicos e incapacidade de conciliar trabalho e família.

Os resultados da pesquisa mostram especificidades na avaliação do crescimento por empreendedoras, evidenciando obstáculos e estímulos ao crescimento, importantes a serem compreendidos (COSTIN, 2012; STILL; TIMMS, 2000), sendo associadas ao nível pessoal e ao nível gerencial. Um aspecto a ser ressaltado refere-se à influência do nível de escolaridade das empreendedoras, tanto na avaliação de determinantes, como na avaliação de dificuldades de crescimento. Além disso, a pesquisa mostrou que variações no montante do capital inicial e na idade com que as empreendedoras criaram as empresas não estão correlacionadas com dificuldades ou com determinantes de crescimento, exceto para diversificação de produtos e de serviços, evidenciando que montante do capital inicial ou idade para abertura das empresas não interferiram na avaliação de determinantes e de dificuldades de crescimento.

A principal contribuição teórica desta pesquisa foi abordar o processo de crescimento além de intenções de crescimento ou de variações, apontando dificuldades e determinantes de crescimento para empreendedoras e mostrando a não influência do capital inicial e da idade no momento da criação da empresa na avaliação de dificuldades e determinantes de crescimento. Ainda que alguns estudos, tal como Roomi et al. (2009) exploraram dificuldades de 
crescimento, eles não analisaram diversas variáveis, não abordando conjuntamente dificuldades $\mathrm{e}$ determinantes de crescimento.

Ademais, as análises de clusters para dificuldades e para determinantes de crescimento mostraram que empreendedoras não apresentam um grupo homogêneo, mas que outras características, além do gênero, segregam o segmento de mulheres empreendedoras.

Uma das limitações desta pesquisa foi a de ter abordado o fenômeno do crescimento em apenas duas dimensões: determinantes e dificuldades de crescimento. O processo do crescimento pode ainda ser explicado por outras dimensões, sugestões para estudos futuros. Outra limitação foi a realização da pesquisa em apenas um setor de atividade. Estudos futuros podem explorar esses elementos em outros setores de atividades, principalmente naqueles em que a inserção de empreendedoras ainda é reduzida.

\section{Referências}

ACHTENHAGEN, L.; NALDI, L.; MELIN, L. Business growth - do practitioners and scholars really talk about the same thing? Entrepreneurship Theory and Practice, 34, 2, p. 289 - 316, 2010.

ALSOS, G.; A. ISAKSEN, J.; LJUNGGREN, E. L. New venture financing and subsequent business growth in men- and women-led businesses.

Entrepreneurship Theory and Practice, 30 (5), p. $667-686,2006$.

ARROYO, M. R.; FUENTES, M., D M.; JIMENÉZ, J.M. R. (2016). Um estudo internacional sobre os fatores que explicam a expectativa de alto crescimento em novos empreendimentos: uma perspectiva de gênero. Revista Brasileira de Gestão de Negócios, 18, 60, p. 171-190, 2016.

BAUGHN, C. C.; CHUA, B.; NEUPERT, K. E. The normative context for women's participation in entrepreneurship: A multi country study.

Entrepreneurship Theory and Practice, 30, 5, p. $687-708,2006$.

BARON, R.; SHANE, S. Empreendedorismo Uma visão do Processo. São Paulo: Thomson, 2007.
BOGREN, M.; FRIEDERICHS, Y.; RENNEMO,

O.; WIDDING, O. Networks women entrepreneurs:

Fruitful to Business Growth? International Journal Of Gender and Entrepreneurship, 5, 1, p. 60-77, 2013.

BOMFIM, L.C.S.; TEIXEIRA, R.M.

Empreendedorismo Feminino: Desafios enfrentados por empreendedoras na gestão de pequenos negócios no setor de Turismo. Revista Pensamento contemporâneo em Administração. Rio de Janeiro, 9, 2, p. 48-69, 2015.

BRITO, L. A. L.; VASCONCELOS, F., C. The Variance Composition of Firm Growth Rates. BAR Brazilian Administration Review, 6, 2, p. 118-136, 2009 .

BRUIN, A.; BRUSH, C. G.; WELTER, F. Advancing a Framework for Coherent Research on Women's Entrepreneurship. Entrepreneurship Theory and Practice, 31, 3, p. 323 - 339, 2007.

BRUSH, C. G. Women-owned businesses: Obstacles and opportunities. Journal of Developmental Entrepreneurship, 2, 1, p. 1 - 24, 1997.

BULLANOVA. O., IKSADEN, E. J.;

KOLVEREID, L. Growth aspirations among women entrepreneurs in high growth firms. Baltic Journal of Management, 11, 2, p. 187-206, 2016.

CHABAUD, D.; NGIJOL, J. La contribution de la théorie des réseaux sociaux à la reconnaissance des opportunités de marché. Presses de l'Université du Québec. Revue Internationale PME, 18, 1, p. 29 46, 2005.

CLARKE, J.; HOLT, R.; BLUNDEL, R. Reimagining the growth process: (Co)-evolving metaphorical representations of entrepreneurial growth. Entrepreneurship \& Regional Development An international Journal. 26, 3-4, p. 234-256, 2014.

COAD, A.; TAMVADA, J. P. Firm growth and barriers to growth among small firms in India. Small Business Economics, 39, p. 383-400, 2012.

COSTIN, Y. In pursuit of growth: an insight into the experience of female entrepreneurs. International Journal of Gender and Entrepreneurship, 4, 2, p. 108-127, 2012. 
DALBORG, C.; VON FRIEDERICHS, Y.;

WINCENT, J. Beyond the numbers: qualitative growth in women's businesses. International Journal of Gender and International

Entrepreneurship. 4, 3, p. 289-315, 2012.

DALBORG, C. The Life cycle in women-owned businesses: from a qualitative growth perspective. International Journal of Management and Entrepreneurship 7, 2, p. 126-147, 2015.

DAVIDSSON, P.; ACHTENHAGEN, L.; NALDI, L. Small Firm Growth. Foundations and Trends ${ }^{\circledR}$ in Entrepreneurship, 6, 2, p. 69 - 166, 2010.

DELMAR, F.; DAVIDSSON, P.; GARTNER, W. Arriving at the high-growth firm. Journal of Business Venturing, 18, 2, p. 189-216, 2003.

DOBBS, M.; HAMILTON, R. T. Small business growth: Recent evidence and new directions. International Journal of Entrepreneurial Behaviour and Research, 13, 5, p. 296 - 322, 2007.

DOLINSKY, A. L.; CAPUTO, R. K. Health and female self-employment. Journal of Small Business Management, 41, 3, p. 233 - 241, 2003.

FLECK, E.; HEGARTY, C.; NEERGAARD, H. The politics of gendered growth. International Journal of Gender and Entrepreneurship, 3, 2, p. 164-173, 2011.

FREEL, M. S.; ROBSON, J. A. Small firm innovation, growth and performance: evidence from Scotland and Northern England. International Small Business Journal, 22, 6, p. 561 - 575, 2004.

GANESAN, R.; KAUR, D.; MAHESHWARI, D. Women entrepreneurs: problems and prospects. The Journal of Entrepreneurship, 11, 1, p. 75 - 93, 2002.

GREENE, P. G.; HART, M. M.; GATEWOOD, E. J.; BRUSH, C. G.; CARTER, N. M. Women entrepreneurs: moving front and center: An overview of research and theory. Coleman White Paper Series, 2003. Disponível em http:/ / usasbe. org/knowledge/whitepapers/greene2003.pdf, acesso em 23 dez 2009.

GUNDRY, L. K.; WELSCH, H. The ambitious entrepreneur: High growth strategies of womenowned enterprises. Journal of Business Venturing, 16, 5, p. $453-470,2001$.
HAIR JR., J. F.; BLACK, W. C.; BABIN, B. J.; ANDERSON, R.E.; TATHAM, R. L. Análise Multivariada de dados. Porto Alegre: Bookman, 2009.

HERMANS, J.; VANDERSTRAETEN, J.; DEJARDIN, M.; RAMDANI, D.; STAM, E.; VAN WITTELOOSTUIJN, A. Ambitious entrepreneurship: Antecedents and consequences. University of Antwerp, Faculty of Applied Economics, Research paper 2012-023, oct 2012. 28 p, 2012.

JULIEN, P. A. Empreendedorismo Regional e a Economia do Conhecimento. São Paulo: Saraiva, 2010.

LEITCH, C.; HILL, F.; NEERGAARD, H.

Entrepreneurial and business growth and the quest for a "comprehensive theory": tilting at windmills? Entrepreneurship Theory and Practice, 34, 2, p. $249-260,2010$.

LERNER, M.; ALMOR, T. Relationships among strategic capabilities and the performance of womenowned small ventures. Journal of Small Business Management, 40, 2, p. 109 - 125, 2002.

MACHADO, H. P. V.; JESUS, M. F. (2010). Efeitos da participação de mulheres em redes sociais: um estudo em um conselho de mulheres executivas. Revista Economia \& Gestão, 10, 24, p.9 - 27, 2010.

MACHADO, H. P. V.; GAZOLA, S.; FABRICIO, J. S.; ANEZ, M. Mulheres Empreendedoras: Razões e Dificuldades para Criação de Empresas. Revista de Administração da Mackenzie, 17, 3, p. 15-38, 2016.

MARLOW, S.; PATTON, D. All Credit to Men? Entrepreneurship, Finance, and Gender. Entrepreneurship Theory and Practice, 29, 6, p. $717-735,2005$.

MARTINS, C. B.; CRNKOVIC, L. H.; PIZZINATTO, N. K.; MACCARI, E. A. (2010). Empreendedorismo Feminino: características e perfil de gestão em Pequenas Empresas. Revista de Administração da UFSM, 3, 2, p. 288-302, 2010.

MCKELVIE, A.; WICKLUND, J. Advancing firm growth research: a focus on growth mode instead of growth rate. Entrepreneurship Theory and Practice, 34, 2, p. $261-288,2010$. 
MITCHELMORE, S.; ROWLEY, J. Growth and planning strategies within women-led SMEs.

Management Decision, 51, 1, p. 83-96, 2013.

MORRIS, M. H., MIYAHASI, N. N., WATTERS, C.E.; COOMBES, S. The Dilemma of Growth: Understanding Venture Size Choices of Women Entrepreneurs. Journal of Small Business Management, 44, 2, p. 221-244, 2006.

ORHAN, M. Women Business Owners in France: The issue of Financing Discrimination. Journal of Small Business Management, 39, 1, p. $95-102$, 2001.

ORSER, B. J.; HOGARTH-SCOTT, S.; RIDING, A. L. Performance, firm size, and management problem solving. Journal of Small Business Management, 38, 4, p. 129 - 146, 2000.

REICHBORN-KJENNERUD, K.; SVARE, H. Entrepreneurial Growth strategies: the female touch. International Journal of Gender and Entrepreneurship, 6, 2, p. 181-199, 2014.

REIS, E. Estatística Multivariada Aplicada. Lisboa: Silabo, 1997.

ROOMI, M. A., HARRISON, P.; BEAUMONTKERRIDGE, J. Women-owned small and medium enterprises in England analysis of factors influencing the growth process. Journal of Small Business and Enterprise Development, 16, 2, p. 270 - 288, 2009.

SANTOS, J. B.; BRITO, L. A. L. Toward a Subjective Measurement Model for Firm Performance. BAR, Brazilian Administration Review, 9, p. 95-117, 2012.

SEBRAE (Org.). Anuário do Trabalho na Micro e Pequena Empresa 2014. São Paulo, $7^{a}$ ed, 2015.

SHANE, S.; VENKATARAMAN, S. The Promise of Entrepreneurship as a field of research. Academy of Management Review, 25, 1, p. 217-226, 2000.

SHELTON, L M. Female Entrepreneurs, WorkFamily Conflict, and Venture Performance: New Insights into the Work-Family Interface. Journal of Small Business Management, 44, 2, p. 285 - 297, 2006.

SINDVEST. Acesso em http: www.sindvestmaringa. com.br, recuperado em 12, dezembro, 2009.
STILL, L. V.; TIMMS, W. Women's business: the flexible alternative work style for women. Women in Management Review, 15, 5/6, p. 272-283, 2000.

STOREY, D. J. Understanding the Small Business Sector. London-Boston: International Thomson Business Press, 1994.

VENUGOPAL, V. Investigating women's intention for entrepreneurial growth. International Journal of Gender and Entrepreneurship, 8, 1, p. 2-27, 2016.

WIKLUND, J.; PATZELT, H.; SHEPHERD, D. A. (2009). Building an integrative model of small business growth. Small Business Economics, 32, 4, p. 351-374, 2009.

WINN, J. Women Entrepreneurs: Can We Remove the Barriers? International Entrepreneurship and Management Journal, 1, p. 381-397, 2005. 